\title{
Miranda
}

Revue pluridisciplinaire du monde anglophone /

Multidisciplinary peer-reviewed journal on the English-

speaking world

$23 \mid 2021$

Modernist Exceptions

\section{Andy Auckbur, Lire la nature dans Arcadia de Sir Philip Sidney : une esthétique du détail}

\section{Raphaëlle Costa de Beauregard}

URL : https://journals.openedition.org/miranda/41633

DOI : 10.4000/miranda.41633

ISSN : 2108-6559

\section{Éditeur}

Université Toulouse - Jean Jaurès

\section{Référence électronique}

Raphaëlle Costa de Beauregard, «Andy Auckbur, Lire la nature dans Arcadia de Sir Philip Sidney : une esthétique du détail », Miranda [En ligne], 23 | 2021, mis en ligne le 11 octobre 2021, consulté le 29 novembre 2021. URL : http://journals.openedition.org/miranda/41633 ; DOI : https://doi.org/10.4000/ miranda.41633

Ce document a été généré automatiquement le 29 novembre 2021.

\section{(c) $(1)$}

Miranda is licensed under a Creative Commons Attribution-NonCommercial-NoDerivatives 4.0 International License. 


\title{
Andy Auckbur, Lire la nature dans Arcadia de Sir Philip Sidney: une esthétique du détail
}

\author{
Raphaëlle Costa de Beauregard
}

\section{RÉFÉRENCE}

Auckbur, Andy, Lire la nature dans Arcadia de Sir Philip Sidney : une esthétique du détail, Reims, Presses universitaires de Reims, 2020, ISBN 978-2-37496-118-7

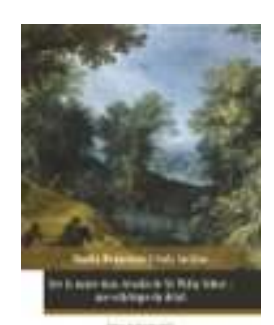

Lire la nature dans Arcadia de Sir Philip Sidney: une esthétique du détail propose une relecture en trois parties du roman de Sidney à la lumière de trois critères : l'univers livresque, l'univers du jardin, et l'univers maniériste. L'ouvrage est accompagné de 10 pl. illustrées en noir et blanc, soulignant ainsi la place accordée au domaine visuel contemporain tout au long de la réflexion. Après une préface du Professeur Christine Sukic, une liste des sigles et abréviations, une note au lecteur qui précise les éditions de Arcadia consultées et citées suivies d'une Introduction générale au contenu de l'œuvre, permettent au lecteur de se repérer plus aisément. À la suite d'une Conclusion générale à l'étude menée, intitulée 'Une grande œuvre et de petits détails', on consultera également au fil de la lecture les annexes qui accompagnent le texte : une bibliographie de 22 pages avec de nombreuses rubriques, les remerciements et enfin les crédits et la table des illustrations, suivis d'une table des matières. (Dans notre compte-rendu, 
seules les références à The Countesse of Pembrokes Arcadia (1593), éditée par Penguin en 1977, sont données, sous la forme N.A. pour New Arcadia, celle utilisée par Andy Auckbur).

Dans le chapitre I, intitulé 'L'univers livresque' (25-106), l'auteur se propose de « mettre en évidence le processus par lequel la richesse de l'univers esthétique de l'auteur [Sidney] s'invite dans la matière de l'œuvre, notamment à travers un art du détail » (20). Ce chapitre se divise en quatre parties : Gravures et traces, Le Livre de la nature, Une nature littéraire, Éloge de la littérature, développant la thèse de l'auteur, à savoir la co-présence du thème de la nature apportant une contribution de l'imaginaire visuel à la mise en forme de l'écriture chez Sidney. Or dans une représentation d'une quête de valeurs telles que le bonheur et l'amour par une fiction conçue comme un ensemble de récits imbriqués et de narrateurs et narratrices diverses, l'univers esthétique du roman serait fondé sur une conviction de l'importance de la présence de la nature, devenue elle-même un «livre des sentiments humains » (47). Une étude du détail de la gravure sur la page de titre de The Countesse of Pembrokes Arcadia (1593) nous invite à en imaginer la présence permanente dans l'imaginaire culturel commun à l'auteur et au lecteur, par le bestiaire, les personnages allégoriques, et les ornements (Fig.1). Dès cette première analyse, nous sommes invités à nous représenter la nature à travers diverses formes visuelles, soit directement, soit par leur évocation littéraire, ou encore dans les devises des personnages, comme autant de digressions et de divertissements à l'intention du lecteur. La nature est donc un livre, lieu commun de la laïcisation du texte sacré (45), dont s'empare Sidney à des fins de réécritures, entre les figures de rhétorique telles que le chiasme, et les modulations des diverses connotations symboliques. Pour étayer cette idée, Andy Auckbur propose une étude du motif de l'arbre par lequel les divers récits imbriqués contribuent à la cohérence esthétique de l'œuvre (82-93). Tout d'abord comme livre, comparé à la gravure sur la pierre, (36-41), puis comme des « signes d'un livre de l'esprit » (51), l'arbre est aussi un motif emprunté aux livres d'emblèmes, faisant ainsi appel à l'intertextualité comme forme d'écriture. Le motif de l'eau est également très présent (68-82), permettant entre autres au sens littéral d'une scène de pêche à la ligne de se lire également au figuré.

Le chapitre II intitulé 'L'univers du jardin' (109-146) se divise en deux parties : L'art des jardins, Le goût des merveilles, toutes les deux consacrées aux liens entre l'art des jardins et l'écriture littéraire du roman. Rappelant que Sidney était un familier des jardins de Penshurst et de Wilton, Andy Auckbur montre comment le roman développe les trois éléments du jardin de la Renaissance, à savoir l'eau, la rivalité entre les arts, et surtout le lien entre l'art du jardin et l'écriture littéraire. L'idée essentielle développée dans ces pages est que le jardin de la Renaissance imite la littérature et que d'autre part, Arcadia imite l'art des jardins (113). L'élément central de cette porosité entre nature cultivée et littérature est la pergola, ou tonnelle, jardin dans le jardin, par exemple le jardin de Kalander (N.A., 73), la maison d'été de Basilius (N.A., 148), ou encore « a fair field appointed for the shepherdish pastimes " (N.A., 175) : dans tous les cas, la tonnelle joue sur le jeu de mots entre 'natural ornaments' et 'artificial inventions.' Particulièrement intéressantes sont les pages consacrées au rôle de la nature 'sauvage', une suite d'arbres et de fourrés devenant pour le promeneur un décor de théâtre lorsque son attention est attirée par un son (une voix humaine dans les exemples cités), dont la source est masquée. Le découpage du lieu en micro-scènes de théâtre (N.A., 295), comme autant de jardins, permet aussi d'opposer des personnages emblématiques, à savoir le courtisan et le paysan. Le motif de la greffe joue ici un rôle 
important, lorsque cet art du jardinier est évoqué et ensuite repris par des personnages, comme si le roman s'inspirait de cet art pour ses développements en micro-récits. Comme l'écrit Auckbur, «chez Sidney, la greffe, en tant qu'activité à travers laquelle l'homme agit sur la nature, est investie d'une dimension civilisatrice qui s'oppose à la violence du monde de la cour » (136). Ainsi, la greffe est-elle aussi une caractéristique de l'art littéraire de Sidney dans Arcadia, permettant une évocation de la beauté humaine en lien avec cette nature. Par exemple un poème décrit le personnage d'Urania en ces termes: "With length laid down, she deck'd the lonely place./ Proud grew the grass that under her did grow/The trees spread out their arms to shade her face ...» (N.A., 200). Urania est appuyée sur un coude, motif employé en peinture qui «donne lieu à des ondulations du corps» (141). D'autres passages accordent une place à la peinture comme reflet dans l'eau, voire une miniature (N.A., 186-187), et apportent des arguments à la thèse de l'auteur qui établit un lien avec le maniérisme, objet du chapitre III.

4 Le chapitre III intitulé 'L'univers maniériste' (149-203) comporte cinq parties: Les courbes du langage, L'art et la nature, Un bijou littéraire, Art et artifice, Un art du détail. Les différentes acceptions du concept d'esthétique maniériste sont utilisées dans cette troisième partie de l'ouvrage de manière à construire un fil conducteur entre des moments différents de notre lecture de l'œuvre de Sidney. Il s'agit d'abord de retrouver une esthétique de la courbe dans la syntaxe, les jeux de mots, voire les blasons du corps féminin. Par exemple, la périphrase fournit un schéma répété tout au long de l'œuvre, comme dans le cas d'un passage au début du livre où "la conclusion résulte d'une miniaturisation de la partie de la phrase qui lui précède » (157). Citons le texte : « As for the houses of the country - for many houses came under their eye - they were all scattered [...] : a show, as it were, of an accompanable solitariness and of a civil wildness » (N.A., 70). D'autres figures de rhétorique telles que le chiasme, ou encore l'hyperbate, introduisent également des sinuosités dans la syntaxe qui forcent le lecteur à en admirer la forme ornementale per se. De sorte que les références aux autres arts telles que la peinture ou la sculpture leur font écho par leur esthétique visuelle. Auckbur étudie en particulier une galerie de portraits (N.A. 158-159) par lesquels le narrateur dépeint l'entrée en scène de chevaliers portant un portrait de la dame qu'ils vont défendre dans un tournoi. Chaque portrait féminin se caractérise par un retournement au cœur de l'éloge de la beauté, qui serait selon Auckbur une « anomalie anatomique » (158), c'est-à-dire un écart par rapport au canon classique de l'harmonie entre le détail et l'ensemble, en particulier en ce qui concerne les proportions (160). Un autre domaine où le maniérisme se révèle est celui du détail emprunté à un domaine spécifique de l'art, tel l'art de l'émail utilisé dans la description pour faire apparaître une nouveauté dans la beauté de la nature, qui serait de l'ordre de l'ornement.

5 Plus significatif encore de la sensibilité de Sidney aux nouvelles formes d'art est son intérêt pour le paysage, art encore à ses débuts (169). «Sidney partageait l'intérêt que les théoriciens de la peinture accordaient au fonctionnement de l'optique et à la diffusion de la lumière » (170). Auckbur rappelle les exposés théoriques d'Alberti tout comme ceux de Léonard en matière de paysages, et une utilisation de considérations empruntées à la peinture telles que les ombres et les lumières.

6 Autre domaine où transparaît le goût maniériste de Sidney, celui de l'orfèvrerie et du langage des pierres permettent là encore, selon Auckbur, d'attirer l'attention du lecteur par la focalisation sur un détail (175). La question du symbolisme des détails est 
abordée par l'analyse de passages consacrés à une description d'un détail du costume d'un personnage (179-18), un ruban blanc dans les cheveux, ou encore un rubis sur la tiare de Musidorus. Le costume de ce prince et celui de Pyrocles sont ainsi investis de valeur symbolique au même titre que celui des héroïnes (182-183).

7 Sidney exploite également le lien entre la description d'un détail dans la nature et la chevelure d'un personnage (188) qui renouvelle la tradition pétrarquiste et, comme le montrent les analyses d'autres passages du roman, se réapproprie certaines descriptions du Tasse dans sa Jérusalem délivrée (196).

Il s'agit donc de définir ce qui serait la 'maniera' de Sidney dans son roman. En réponse aux principes esthétiques déjà introduits auprès du lecteur éduqué de son temps tels que l'idéal de la grâce définit par Castiglione, la sprezzatura (189), et l'idéal de beauté du Canzoniere, Sidney fait des références originales à la peinture. Ainsi «le principe de dissolution des contours rappelle l'approche chromatique des peintres vénitiens de la Renaissance » (196), permettant une « poétique de l'enrichissement mutuel » (196) dans des descriptions de micro-scènes semblables à des miniatures. Auckbur cite à titre d'exemple (196) la description du cou de Parthenia qui apparaît une fois délivrée de son heaume (N.A.528). Plus intéressante encore est l'analyse de la description en termes picturaux par Sidney de son héroïne Pamela retenue prisonnière et occupée à une broderie (198-202).

9 Telles sont les conclusions auxquelles Auckbur nous conduit (207-211). L'esthétique du détail, et son inscription dans celle de la peinture en petit par la fréquente insertion d'un effet de cadrage, participent à un "maillage complexe de références littéraires » (208). L'évocation de la nature, en parallèle à cette intertextualité subtile, permet à un ensemble d'images de s'immiscer dans la mémoire du lecteur «par lesquelles il pourra envisager le monde réel à travers le prisme de formes nouvelles » (209). Plus encore, le lecteur peut se constituer "un univers esthétique et vivre au quotidien l'expérience sidnéenne de la beauté » (211).

Cette étude qui se propose de lire la nature dans le roman de Sir Philip Sidney a le mérite de nous inviter à relire le texte, sinon dans son entier, du moins dans de longs passages. Car relus ainsi avec une attention particulière à l'écriture, ces passages choisis par Auckbur en éclairent non seulement l'originalité mais aussi, sans doute, les raisons pour lesquelles l'ouvrage a pu exercer une telle influence culturelle et littéraire. Moins appréciée au XIXe siècle, l'œuvre a connu un regain d'intérêt grâce à l'essai de Virginia Woolf de 1925, dans lequel elle compare ce texte à « un globe lumineux où se reflètent les germes de toute la fiction anglaise » (20), comme nous le rappelle Andy Auckbur dans son Introduction. C'est bien en effet de certains de ces «germes » dont il est question de manière détaillée dans son ouvrage.

\section{INDEX}

Keywords : nature, art, aesthetics, writing, garden, mannerism

Mots-clés : nature, art, esthétique, écriture, jardin, maniérisme 
AUTEURS

RAPHAËLLE COSTA DE BEAUREGARD

Professeur émérite

Université Toulouse Jean Jaurès

costa_de_beauraph@orange.fr 